\title{
Study on Robotic Seam Tracking of Laser Scanning System
}

\author{
Bin Zhang \\ China JiLiang University \\ College of Metrology and Measurement \\ Hangzhou, China \\ azhwwbin@163.com
}

\author{
Chen Tang \\ China JiLiang University \\ College of Metrology and Measurement \\ Hangzhou, China \\ let_us_shine@126.com
}

\begin{abstract}
A kind of laser scanning weld seam tracking system is studied. The calibration method for the system parameters is presented and the recognition method for the seam characteristic point is given based on digital image processing. Firstly, preprocess the laser image using median filter method, adaptive threshold method and isolated point filter method and find the seam characteristic point using the digital morphological method. Then, accomplish the parameter calibration of the structured light according to laser plane infinity point projection in the camera obtained by moving the sensor with the target of a set of parallel lines. Finally, Change the position of the robot to detect fixed target and solve the robot hand-eye matrix. The calibration process of the sensor is convenient and the precision is reliable. $U$ weld seam on the spatial free-form surface can be measured by the sensor.
\end{abstract}

Keywords-digitalmorphological; hand-eye matrix; seam tracking; triangulation; laser calibration

\section{INTRODUCTION}

In order to meet the needs of modern industrial production, the application of robot in welding has become the hot spot of research. The main application of the sensor includes the arc sensor [1] and the visual sensor [2]. Along with the development of computer image processing technology [3], the use of visual sensors is more extensive. The laser scanning welding seam tracking sensor used in this system is a kind of visual sensor.

The sensor transmits the two dimensional images information to the computer. So as to obtain three dimensional information in the weld robot base coordinate system, calibration for laser sensors and robot hand-eye is necessary. Calibration of the sensor including camera and structure light calibration, the technology of camera calibration [4] is relatively mature. The earliest method of calibration of laser structured light is tooth shape calibration [5] and wire drawing calibration [6]. These methods utilize the specific calibration rake to generate the target point on the structural light plane. In addition, the coordinate of the target point in the structure plane of the structure is obtained by using the auxiliary measuring instrument. As to robot hand-eye calibration, the most classical algorithm is solved by the equation $\mathrm{AX}=\mathrm{XB}[7$ 8]. Between double adjacent of the robot movement, "A" means transformation matrix at the end of the manipulator, "B" represents sensor's transformation matrix, and " $\mathrm{X}$ " represents the hand-eye relationship. On this basis, there are different algorithms for solving: Qi [9] uses the method of robot handeye calibration based on the linear feature. But in practical applications, a linear feature is difficult to constitute and accuracy is hard to guarantee. $\mathrm{Hu}$ [10] founds another calibration method for robot hand-eye based on planar structure, but it also is existed the problem that is difficult to ensure plane features.

From the perspective of the practical application, the calibration method should be simple and feasible in the premise of ensuring the accuracy of calibration. In this paper, the calibration of structured light is projected on the basis of the infinite point on the laser plane. The target points are detected by the same fixed robot pose to achieve robot handeye's calibration. The entire calibration process does not require a precise calibration target and an auxiliary measuring instrument. Meanwhile, the result is reliable and accurate.

\section{Robotic Seam Tracking of LASER Scanning System}

The robot laser scanning welding seam tracking system is composed of six degrees of freedom robot and sensor. As shown in Fig. 1, the robot controls the laser scanning seam tracking sensor to scan the weld. Then, the video camera transmits the laser image of the weld information to the computer.

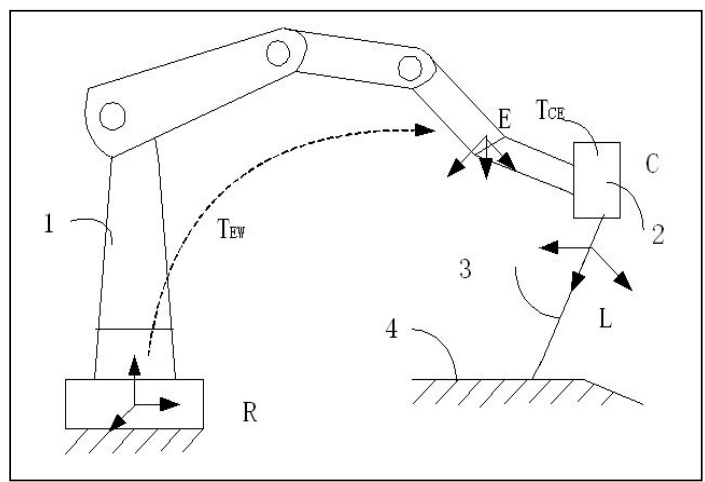

Fig. 1. The robot installed laser sensor.

1-Robot; 2-Laser welding seam tracking sensor; 3- Laser; 4-Seam 
In the figure, $\{R\}$ is a basic coordinate system with the base of the robot; $\{\mathrm{E}\}$ is manipulator end coordinate system; $\{C\}$ is camera coordinate system.

\section{Weld FEATURE PoINT DEtection}

Laser image is captured by the camera's presence arc, spatter, and other noise. According to the characteristics of the original weld image, pretreatment is the interference of the maximum degree of noise removal. In this paper, the laser image preprocessing process is shown in Fig. 2.

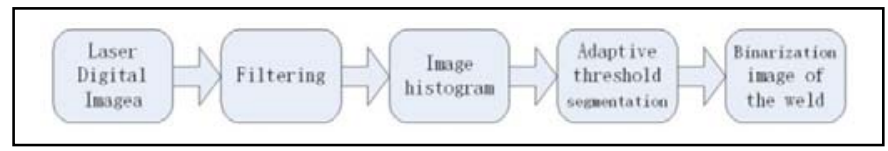

Fig. 2. Laser image preprocessing algorithm schematic.

The following will use U-shaped curved seam image processing as an example. When the depth of the weld change, the image is different. As shown in Fig. 3, (a) is the image of the deep seam in the pre - and after - processing, while (b) is the image of the weld which is more shallow.

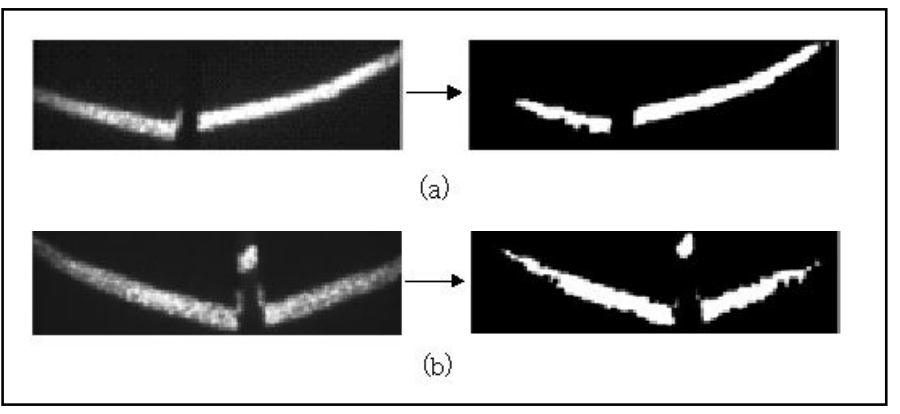

Fig. 3. Laser image after pretreatment

After obtaining a clear image of the weld, first of all, there is using different gray values to show different image of regions. Furthermore, it is filtering out the isolated points and small regions in the image. Finally, the image only exists two laser striped light.

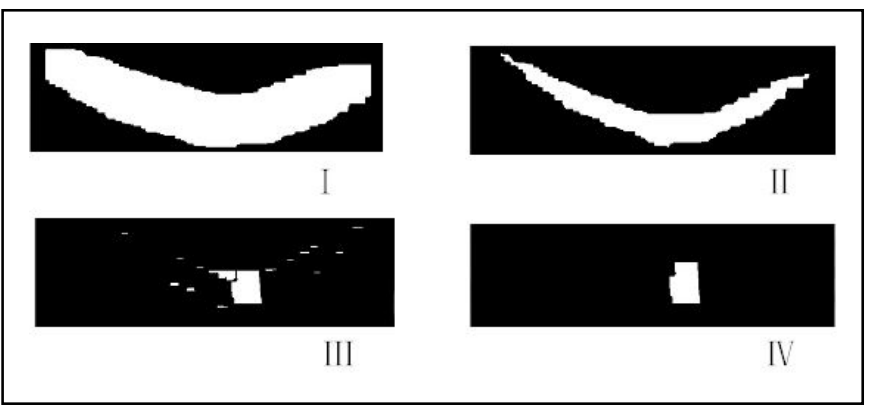

Fig. 4. Weld feature point detection

As shown in Fig. 4, when taking curved weld as an example. The extraction of weld feature points using not only expansion and erosion algorithm in mathematical morphology but image subtraction. Which make the algorithm can be applied to planar and curved surfaces. Specific steps are as follows:

a) The expansion operation of the laser image is firstly carried out, which connects the image of the two laser structured light.

b) The same number of laser images were performed in the same number of times, so the shape of the two laser structured light image was restored to the expansion operation.

c) A reduction operation on the image of the welded joint before the expansion operation of the laser structured light image. Through this operation, the image will emergence a little laser image and lots of isolated areas.

d) Representing different regions of the imageon the basis ofdifferent gray values, then filtering out the isolated points and small regions. In the final image,the weld feature points are obtained.

e) Finding out the center of the figure in laser image, which the weld feature points in the image coordinates.

\section{SYSTEM CALIBRATION}

\section{A. Calibration of Structure Light Sensor}

The sensor uses triangulation method to measure the weld seam. The measurement model used in the system shown as Fig. 5. The process can also be described as a laser light plane by means of projection transformation to a camera's image plane.

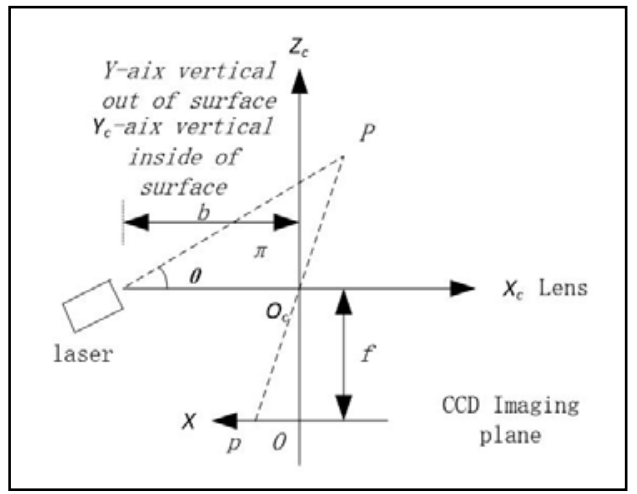

Fig. 5. The measurement model of structured light

In the Fig. 5, PI is the plane projected by laser plane; Oc$\mathrm{XcYcZc}$ is camera coordinate system based on the intersection of the optical axis and lens, O-XY for image coordinate system. If $\mathrm{P}(\mathrm{xc}, \mathrm{yc}, \mathrm{zc})$ is a point in laser plane, its coordinates in the image plane is $\mathrm{p}(\mathrm{x}, \mathrm{y})$. According to the principle of triangulation method, the following relations are obtained:

$$
\frac{x_{c}}{x}=\frac{z_{c}}{f}, \frac{y_{c}}{y}=\frac{z_{c}}{f}, \tan \theta=\frac{z_{c}}{b+x_{c}}
$$

Among these relations, $\mathrm{f}$ is camera focal length; $\mathrm{B}$ is the distance between the laser plane and the Xc axis and the optical axis of the camera which is called "Base length"; $\theta$ is 
the angle between $\mathrm{Xc}$ and laser plane. The conversion relationship between camera coordinate and image coordinate of target point is followed:

$$
\left[\begin{array}{l}
x_{c} \\
y_{c} \\
z_{c}
\end{array}\right]=\frac{b}{f \cot \theta-x}\left[\begin{array}{l}
x \\
y \\
f
\end{array}\right]
$$

If the type of camera focal length $\mathrm{f}$, projection angle, base length B all are known. According to (2), any point in the 3D space can correspond to the points in the 2D image. System internal parameters calibration of laser sensor is to calculate "f", " $\theta$ " and "b".

The parameter " $\mathrm{f}$ " can be obtained by camera calibration. According to the principle of triangulation method:

$$
\left\{\begin{array}{l}
x_{c}=\frac{b \cdot x}{f \cot \theta-x} \\
y_{c}=\frac{b \cdot y}{f \cdot \cot (-x} \\
z_{c}=\frac{f \cdot b}{f \cdot \cot l-x}
\end{array}\right.
$$

If the point $\mathrm{P}$ is in the infinite distance of the laser plane, it means $(\mathrm{xc}, \mathrm{yc}, \mathrm{zc}) \rightarrow \infty, \mathrm{zc} \rightarrow \infty$, so $\mathrm{f} \cdot \cot \theta-\mathrm{x}_{\infty} \rightarrow 0$. Rearranging equation :

$$
\cot \theta=x_{\infty} / f
$$

$\mathrm{X}_{\infty}$ is the image coordinate projected by laser plane at infinity point $\mathrm{P}$ in camera. Make the derivation of the equation $\mathrm{y}_{\mathrm{c}}=\frac{\mathrm{b} \cdot \mathrm{y}}{\mathrm{f} \cdot \cot \theta-\mathrm{x}}$, it will become

$$
\begin{gathered}
\Delta y_{c}=\frac{\partial y_{c}}{\partial y}+\frac{\partial y_{c}}{\partial x} \\
=\frac{b}{f \cot \theta-x} \cdot \Delta y+\frac{\partial y_{c}}{\partial x}
\end{gathered}
$$

If $\Delta x=0$, the equation can be written as follows:

$$
\Delta \mathrm{y}_{\mathrm{c}}=\frac{\mathrm{b}}{\mathrm{f} \cot (-\mathrm{x}} \cdot \Delta \mathrm{y}
$$

On the basis of (6):

$$
b=\frac{f \cot \theta-x}{\Delta y} \cdot \Delta y_{c}
$$

In (7), $X$ is the current image coordinates. If the size of the $\Delta y c$ can be controlled and calculating the $\Delta y$ by image processing, the base length " $\mathrm{b}$ " of the structured light can be determined.

\section{B. Robot Hand-Eye Calibration}

The robot hand-eye calibration matrix is to determine the conversion of camera coordinate and the manipulator coordinate.

As shown in Fig. 1, the $T_{E R}$ is the end of robot hand transition matrix " $E$ " relative to the base coordinate matrix " $R$ ".
From the robot visual controller, spin matrix $\mathrm{R}_{\mathrm{ER}}$ and translation vector $t_{E R}$ can be read directly. $T_{C E}$ is the transformation matrix of camera coordinate system " $\mathrm{C}$ " relative to the end of robot hand transition matrix "E", which includes spin matrix $R_{C E}$ and translation vector $t_{C E}$. Because the sensor is fixed at the end of the manipulator, $T_{C E}$ does not change with the movement of the manipulator.

In this paper, the same target point $M$ is detected by changing the robot's pose. Assuming that the robot is in the "i" pose, the coordinates of the target point $\mathrm{M}$ in the camera coordinate system is $\mathrm{M}_{\mathrm{i}}^{\mathrm{C}}$ and in robot base coordinate system is $\mathrm{M}^{\mathrm{R}}$. The conversion relationship between the two coordinates is as follows:

$$
\begin{gathered}
\mathrm{M}^{\mathrm{R}}=\mathrm{T}_{\mathrm{ER}} \mathrm{T}_{\mathrm{CE}} \mathrm{M}_{\mathrm{i}}^{\mathrm{C}} \\
{\left[\begin{array}{c}
\mathrm{M}^{\mathrm{R}} \\
1
\end{array}\right]=\left[\begin{array}{cc}
\mathrm{R}_{\mathrm{ERi}} & \mathrm{t}_{\mathrm{ERi}} \\
0 & 1
\end{array}\right]\left[\begin{array}{cc}
\mathrm{R}_{\mathrm{CE}} & \mathrm{t}_{\mathrm{CE}} \\
0 & 1
\end{array}\right]\left[\begin{array}{c}
\mathrm{M}_{\mathrm{i}}^{\mathrm{c}} \\
1
\end{array}\right]}
\end{gathered}
$$

Expanding the above equation:

$$
M^{R}=R_{E R i} R_{C E} M_{i}^{c}+R_{E R i} t_{C E}+t_{E R i}
$$

Controlling the robot hand to the target point $\mathrm{M}$ on the weld for many times:

$$
\left\{\begin{array}{c}
M^{R}=R_{E R 1} R_{C E} M_{1}^{c}+R_{E R 1} t_{C E}+t_{E R 1} \\
M^{R}=R_{E R 2} R_{C E} M_{2}^{c}+R_{E R 2} t_{C E}+t_{E R 2} \\
\vdots \\
M^{R}=R_{E R n} R_{C E} M_{n}^{c}+R_{E R n} t_{C E}+t_{E R n}
\end{array}\right.
$$

\section{1) Spin matrix solver}

If the pose of the robot is kept constant during movement, then $\mathrm{R}_{\mathrm{ER} 1}=\mathrm{R}_{\mathrm{ER} 2}=\ldots=\mathrm{R}_{\mathrm{ERn}}=\mathrm{R}_{0}$. Because $\mathrm{R}_{0}$ isan orthogonal matrix, so $R_{0}^{-1}=R_{0}^{T}$. Through multiple measurements:

$$
\begin{gathered}
R_{C E}\left[M_{2}^{c}-M_{1}^{c} M_{3}^{c}-M_{1}^{c} \ldots M_{n}^{c}-M_{1}^{c}\right]=R_{0}^{T} \\
{\left[t_{E R 1}-t_{E R 2} t_{E R 1}-t_{E R 3} \ldots t_{E R 1}-t_{E R n}\right]}
\end{gathered}
$$

Assuming that $\mathrm{A} 1=\left[\mathrm{M}_{2}^{\mathrm{c}}-\mathrm{M}_{1}^{\mathrm{c}} \mathrm{M}_{3}^{\mathrm{c}}-\mathrm{M}_{1}^{\mathrm{c}} \ldots \mathrm{M}_{\mathrm{n}}^{\mathrm{c}}-\mathrm{M}_{1}^{\mathrm{c}}\right]$; $\mathrm{b} 1=\left[\mathrm{t}_{\mathrm{ER} 1}-\mathrm{t}_{\mathrm{ER} 2} \mathrm{t}_{\mathrm{ER} 1}-\mathrm{t}_{\mathrm{ER} 3} \ldots \mathrm{t}_{\mathrm{ER} 1}-\mathrm{t}_{\mathrm{ERn}}\right]$. The equality can be simplified into $R_{C E} A_{1}=b_{1}$. Then using the method of "Singular Value Decomposition" to solve it.

$$
\mathrm{R}_{\mathrm{CE}}=\mathrm{VU}^{\mathrm{T}}
$$

$\mathrm{U}$ and $\mathrm{V}$ are respectively the right singular matrix and the left singular matrix of the matrix " $A_{1} b_{1}^{T}$.

2) Translation vector

According to (11):

$$
\begin{gathered}
\left(R_{E R 2}-R_{E R 1}\right) t_{C E}=R_{E R 1} R_{C E} M_{1}^{c}-R_{E R 2} \\
R_{C E} M_{2}^{c}+t_{E R 1}-t_{E R 2}
\end{gathered}
$$


Controlling of the robot arm to detect the target point, which could make an equation $\mathrm{A}_{2} \mathrm{t}_{\mathrm{CE}}=\mathrm{b}_{2}$. Solving the equation based on least square method:

$$
t_{C E}=\left(A_{2}^{T} A_{2}\right)^{-1} A_{2}^{\mathrm{T}} b_{2}
$$

\section{System Calibration and Welding Seam Tracking EXPERIMENT}

\section{A. Calibration of Sensor}

When the climb up, the plane at infinity is a straight line. Similarly, the two parallel lines in the infinite to a point. As shown in Fig. 6, put calibration target in horizontal level and adjust the position of the sensor to make camera's optical axis is perpendicular to the target. In the same time, laser plane and two parallel lines in A, B two points. Then controlling the camera to move along the optical axis, a series of points named " $\mathrm{A}_{\mathrm{i}}, \mathrm{B}_{\mathrm{i}}(\mathrm{i}=1,2 \ldots \mathrm{n})$ " are generated in laser plane. These points project in the camera are $a_{i}, b_{i}(i=1,2 \ldots n)$. Two parallel lines are formed by "Ai、Bi "on the laser plane, which will become the same point in infinity laser plane.

Unitizing $a_{i}$ and $b_{i}$ to fit straight line. The intersection of the two straight lines is the projection of a point on the camera in the infinity of the laser plane, whose coordinate is $(6.9122$, 0.2598 ). So $x_{\infty}=6.9122 \mathrm{~mm}$. Focal length can be obtained in the camera calibration, $\mathrm{f}=6.7595 \mathrm{~mm}$.

$\cot \theta=\mathrm{x}_{\infty} / \mathrm{f}=1.022$

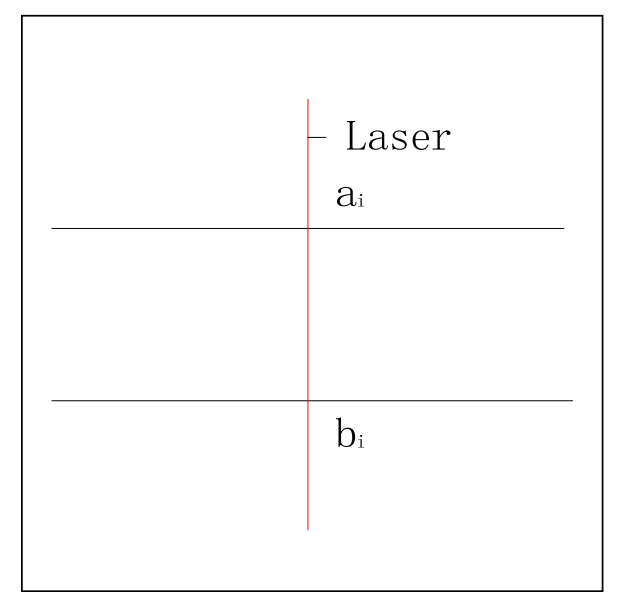

Fig. 6. Calibration harrow.

By moving the sensor changes the size of the Delta $v$ and delta yc, sensor based $b$ can be obtained. The calibration results are shown in Table I

TABLE I. SENSOR INTERNAL PARAMETERS

\begin{tabular}{|c|c|c|c|}
\hline Parameter & $\boldsymbol{f}$ & $\boldsymbol{b}$ & $\boldsymbol{\theta}$ \\
\hline Value & $6.7595 \mathrm{~mm}$ & $91.42 \mathrm{~mm}$ & 44.37 \\
\hline
\end{tabular}

\section{B. Robot Hand-Eye Matrix}

The target point $\mathrm{M}$ was detected with five times pure translational motion and five motion pose, and the end of each manipulator was recorded. As shown in Fig. 7:
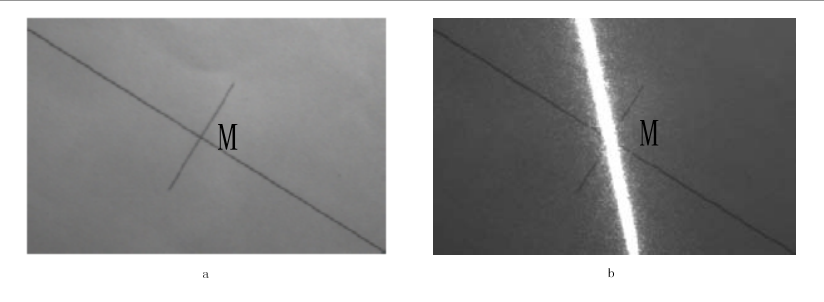

Fig. 7. The calibration target of hand-eye calibration experiments.

TableII is the coordinate of target points in the camera coordinate system and Table III is the pose of the manipulator end of each measurement.

TABLE II. THE CAMERA COORDINATES OF THE TARGET.

\begin{tabular}{|c|c|}
\hline Pose & $\mathbf{M}_{\mathbf{i}}^{\mathbf{C}}$ \\
\hline 1 & $(6.02,-1.706,95.18)$ \\
\hline 2 & $(-1.85,8.09,87.48)$ \\
\hline 3 & $(30.28,19.81,118.90)$ \\
\hline 4 & $(-18.07,6.51,71.61)$ \\
\hline 5 & $(5.49,-9.01,94.66)$ \\
\hline 6 & $(-6.34,-0.21,83.08)$ \\
\hline 7 & $(-3.61,2.31,85.75)$ \\
\hline 8 & $(-3.50,1.14,85.86)$ \\
\hline 9 & $(-2.39,-0.67,86.94)$ \\
\hline 10 & $(-5.21,1.17,84.18)$ \\
\hline
\end{tabular}

TABLE III. Machine HAND TERminAl StANCE

\begin{tabular}{|c|c|}
\hline Pose & end of manipulator posture $(\alpha, \beta, \gamma, \mathrm{t} 1, \mathrm{t} 2, \mathrm{t} 3)$ \\
\hline 1 & $(47.48,-87.44,-134.4,-741.3,-57.73,121.58)$ \\
\hline 2 & $(47.48,-87.44,-134.4,-732.27,-67.67,112.11)$ \\
\hline 3 & $(47.48,-87.44,-134.4,-751.40,-78.13,133.23)$ \\
\hline 4 & $(47.48,-87.44,-134.4,-719.45,-65.73,95.79)$ \\
\hline 5 & $(47.48,-87.44,-134.4,-739.86,-50.61,120.12)$ \\
\hline 6 & $(8.50,-89.22,-171.45,-743.41,-78.31,108.13)$ \\
\hline 7 & $(-106.02,-81.61,63.2,-756.73,-109.62,111.71)$ \\
\hline 8 & $(-109.65,-56.53,62.12,-785.94,-111.82,116.22)$ \\
\hline 9 & $(-171.91,-77.03,-15.71,-777.23,-128.52,110.61)$ \\
\hline 10 & $(121.41,-52.22,-64.42,-788.11,-78.01,117.97)$ \\
\hline
\end{tabular}


What can be seen from TabIII is the Euler angle is shown on the controller. Conversion relationship between Euler angle and transition matrix as followed:

$$
\begin{gathered}
\mathrm{T}_{\mathrm{ER}}= \\
{\left[\begin{array}{cccc}
\cos \beta \cos \gamma & -\cos \beta \sin \gamma & \sin \beta & t_{1} \\
\sin \alpha \sin \beta \cos \gamma+\cos \beta \sin \gamma & \cos \alpha \cos \gamma-\sin \alpha \sin \beta \sin \gamma & -\sin \alpha \cos \beta t_{2} \\
\sin \alpha \sin \gamma-\cos \alpha \sin \beta \cos \gamma & \cos \alpha \sin \beta \sin \gamma+\sin \alpha \cos \beta & \cos \alpha \cos \beta & t_{3} \\
0 & 0 & 0 & 1
\end{array}\right]}
\end{gathered}
$$
data:

According to TableII and TableIII of the first four sets of

$$
\mathrm{R}_{\mathrm{CE}}=\left[\begin{array}{ccc}
0.0022 & -0.0000 & -1.0000 \\
-0.0004 & -1.0000 & 0.0000 \\
1.0000 & 0.0004 & -0.0022
\end{array}\right]
$$

On the basis of the last four set of data:

$$
\mathrm{t}_{\mathrm{CE}}=\left[\begin{array}{lll}
49.8773 & -46.9190 & -16.1010
\end{array}\right]^{\mathrm{T}}
$$

\section{Welding Seam Tracking Experiment}

As shown in Fig. 8, in order to verify the reliability of the system in the seam tracking, a robot laser scanning welding seam tracking system is used to detect the welding seam of the workpiece.

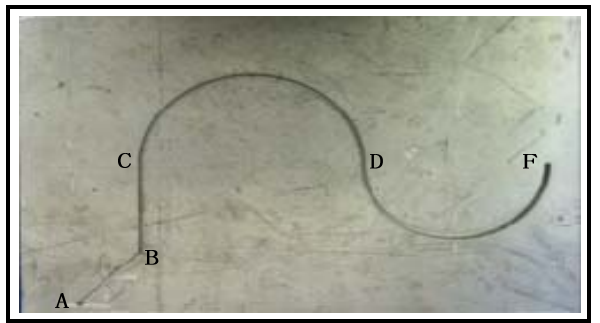

Fig. 8. The experiment platform

Firstly, robot controls the sensor to collect three hundred laser images contained with information of weld one by one. Secondly, using an algorithm to analyse these images. The final Fitting Weld track is shown as Fig. 9.

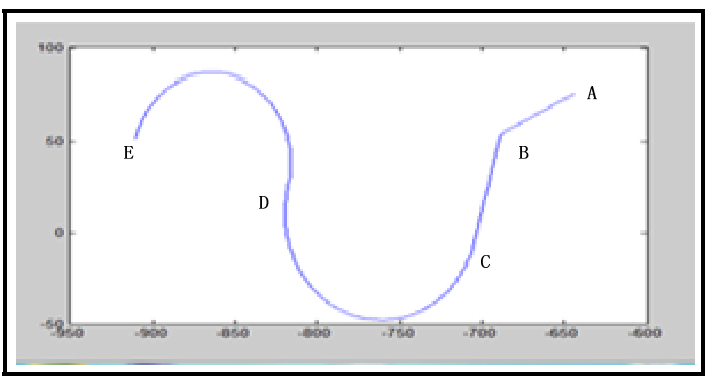

Fig. 9. Seam track

\section{CONCLUSION}

In this paper, the research object of the laser scanning welding seam tracking system and laser image processing based on digital morphology. According to the mathematical model of sensor measurement, the result makes the conclusion about the method of proposed calibration and robot hand eye calibration sensor structure parameters. In future, the method of robot motion control will be further studied on this basis.

\section{REFERENCES}

[1] B. Hong, X. Lai,and F.L. Wei, et al."Seam tracking system with leading magnetic-control arc sensor", Transduc. Microsyst. Tech.vol. 27, pp. 9395, 2008.

[2] J.R. Lin, B. Wu, and P. Gao,et al. "2-D Robot seam tracking system based on structured-light visual sensor", J. of Optoeleetronies: Laser, pp. 793-795, 2009

[3] R.B. Fend, and Z.Y. Xu, "The application of OpenCV on the real-time detection system of the seam"Ind. Instrum. Automat. Dvices, no. 4, pp. $39-42,2012$.

[4] W.J. Gao, and J. Li. "Research on Camera Calibration Technique Based on OpenCV",Comput.Digit.Eng., vol. 36, no. 12, pp. 128-130, 2008.

[5] F.J. Duan, F.G. Liu, and S.H. Ye. "A New Accurate Method for the Calibration of Line Structured Light Sensor", J. Instrument, vol. 21, no. 1, pp. 108-110, 2000.

[6] Z. Gan, and Q. Tang, "Laser stripe sensor calibration", Advanced Topics in Science \& Technology in China, 2011.

[7] Y. Wang, Z.L. Dong, and M.X. Sun, et al."A new approach based robot hand-eye calibration linear prototype", PR and AI, vol. 18, no. 4, pp. 491-495, 2005

[8] L. Li, R.L. Bai, and F. Ji, et al. "Calibration technique for structured light vision guided trajectory tracking system.” Comput. Eng. Appl. vol. 50 , no. 16 , pp. 259-264, 2014

[9] A.Y. Qi, F. Jing, and M. Tan,"Line-feature-based calibration method of structured light plane parameters for robot hand-eye system", Opt. Eng., vol. 52, no. 3, pp. 254-260, 2013.

[10] J.S. Hu, and Y.J.Chang, "Calibration of an eye-to-hand system using a laser pointer on hand and planar constraints", Robotics and Automation (ICRA), 2011 IEEE Int. Conf. IEEE, pp. 982-987, 2011 